\title{
Characteristics of the similarity index in a Korean medical journal
}

\author{
Seunghyun Chung ${ }^{1}$, Jeunghyuk Lee ${ }^{1}$, Younsuk Lee ${ }^{1,2}$, Ha Yeon Park ${ }^{1}$, and \\ Daehwan Kim ${ }^{1}$ \\ ${ }^{1}$ Department of Anesthesiology, Dongguk University Medical Center, ${ }^{2}$ D/M Statistics Institute, Dongguk University, \\ Goyang, Korea
}

Background: Journal editors have exercised their control over submitted papers having a high similarity index. Despite widespread suspicion of possible plagiarism on a high similarity index, our study focused on the real effect of the similarity index on the value of a scientific paper.

Methods: This research examined the percent values of the similarity index from 978 submitted ( 420 published) papers in the Korean Journal of Anesthesiology since 2012. Thus, this study aimed to identify the correlation between the similarity index and the value of a paper. The value of a paper was evaluated in two distinct phases (during a peer-review process vs. after publication), and the value of a published paper was evaluated in two aspects (academic citation vs. social media appearance).

Results: Yearly mean values of the similarity index ranged from $16 \%$ to $19 \%$. There were 254 papers cited at least once and 179 papers appearing at least once in social media. The similarity index affected the acceptance/rejection of a paper in various ways; although the influence was not linear and the cutoff measures were distinctive among the types of papers, both extremes were related to a high rate of rejection. After publication, the similarity index had no effect on academic citation or social media appearance according to the paper.

Conclusions: The finding suggested that the similarity index no longer had an influence on academic citation or social media appearance according to the paper after publication, while the similarity index affected the acceptance/rejection of a submitted paper. Proofreading and intervention for finalizing the draft by the editors might play a role in achieving uniform quality of the publication.

Key Words: Bibliometrics, Literature based discovery, Peer review, Plagiarism, Similarity index, Social media.

Corresponding author: Younsuk Lee, M.D., Ph.D.

Department of Anesthesiology, Dongguk University Medical Center, D/M Statistics Institute, Dongguk University, 27, Dongguk-ro, Ilsandong-gu, Goyang 10326, Korea

Tel: 82-31-961-7872, Fax: 82-31-961-7864

Email: dryl@icloud.com

ORCID: http://orcid.org/0000-0003-2488-5926

Received: January 3, 2017.

Revised: January 16, 2017.

Accepted: January 31, 2017.

Korean J Anesthesiol 2017 June 70(3): 327-334

https://doi.org/10.4097/kjae.2017.70.3.327

\section{Introduction}

High phrasal similarity, usually represented by a high percentage of the matching text or a high percentage of the similarity index, has become one of the most perplexing issues for authors, reviewers, and editors working in the medical field worldwide. Authors and editors are afraid of being suspected of plagiarism without any respect to their intentions. As some editors [1] have pointed out that increasing plagiarism obliges research institutes and journals to take measures against text-theft through the internet, and dedicated web-based applications have been widely used to estimate the similarity index. The Korean Journal of Anesthesiology (KJA) has introduced a new process where all of the submitted papers must undergo an estimation

(c) This is an open-access article distributed under the terms of the Creative Commons Attribution Non-Commercial License (http://creativecommons.org/ licenses/by-nc/4.0/), which permits unrestricted non-commercial use, distribution, and reproduction in any medium, provided the original work is properly cited. 
of the similarity index since April 2012. The similarity index is a percent score estimating how many phrases of a manuscript are identical to those in a previously published work. Some medical journal editors have closed their submission gate to a manuscript having a high similarity index, by equipping with a filtration step prechecked by a computer-driven system. Still, some of our in-house editors have claimed that they could hardly find any value in papers having a high similarity index. However, others have commented that a high similarity index would be inevitable according to the research discipline or the nature of research itself. Although the percentage of the similarity index has become a standard measure against plagiarism among authors and editors working in the medical writing field, its characteristics, particularly those affecting the value of a paper, have not been investigated. To identify the correlation between the similarity index and the value of a paper, we employed 'probability to be accepted,' 'academics cited-by frequency', and 'appearance in social media.' More specifically, the research aimed to answer the following questions:

- Does a submitted paper with a high phrasal similarity have more chance to get rejected?

- Does a published paper with a high phrasal similarity have a lower chance to attract citation?

- Does a published paper with a high phrasal similarity appear less frequently in social media?

The goal of our research was to eradicate a previous insight that a submitted paper having a high similarity index might hardly have any value. We did not mention about plagiarism or insincere writing throughout the manuscript. Instead, we depict how the similarity index appears, how it affects the decision of a peer review, and how editors are confident about the insights independent of the similarity index.

\section{Materials and Methods}

The authors were permitted to handle the datasets extracted from the online KJA submission system be used in this research at the 62nd Meeting of The Research/Publication Ethics Committee of the KJA Editors (26 January, 2016).

\section{Construction of datasets}

We analyzed two datasets retrieved from the online KJA submission/review system. The first dataset $(n=978)$ comprised research reports (including clinical and experimental investigations) and case reports submitted from 2012 to 2015. Papers under peer review were excluded. The first dataset contained the following 5 data fields:

- Submission year: 2012-2015

- Type of the paper: A research report (including a clinical investigation and an experimental investigation) and a case report

- Similarity index: Estimated using the iThenticate Plagiarism Detection Program

- Decision: Accepted/Rejected (Rejection included withdrawal by the author.)

- DOI: Digital object identifier for a published paper.

The similarity index percent (iThenticate Plagiarism Detection Program, Turnitin, Oakland, United States) provided a measure of phrasal similarity. The similarity index was estimated with a set of option variables such as the exclusion of bibliography, and the inclusion of matches over 6 consecutive words and that of $1 \%$ or greater total match percentage per source. As an excerpt from the first dataset, the second dataset $(n=420)$ comprised papers published by the KJA.

According to the context of the paper, we classified the value of a paper into the following two categories: the value of a submitted paper and the value of a published paper. 'Probability to be accepted' played a role in indicating the value of a submitted paper, and for a published paper, the value was subclassified into two aspects such as academic citation and social media appearance.

The cited-by frequency measures were collected manually from the websites of Scopus and Web of Science (WoS), and by using program-driven application programming interface (API) calling to CrossRef. These measures were combined into the second dataset through the DOI as a key field. Appearance in social media, such as Facebook public wall post and Twitter, and Altmetric.com overall score were retrieved by using Altmetric.com open API calling. Web-retrieval and API calling were performed on February 10, 2016. The second dataset contained the following 10 data fields:

- Publication year: 2012-2015

- Type of the paper: A research report and a case report

- Similarity index

- Cited-by frequency

- CrossRef

- Scopus

- WoS

- Social media appearance

- Altmetric.com overall score

- Facebook public wall post

- Twitter

- DOI

One hundred four records of the dataset had missed the similarity index, which might have resulted from a clerical error or an earlier submission before April 2012, when the KJA had introduced measurement of the similarity index. Thus, we employed 420 records for estimating summary statistics and 316 records for estimating the effect of the similarity index on the 


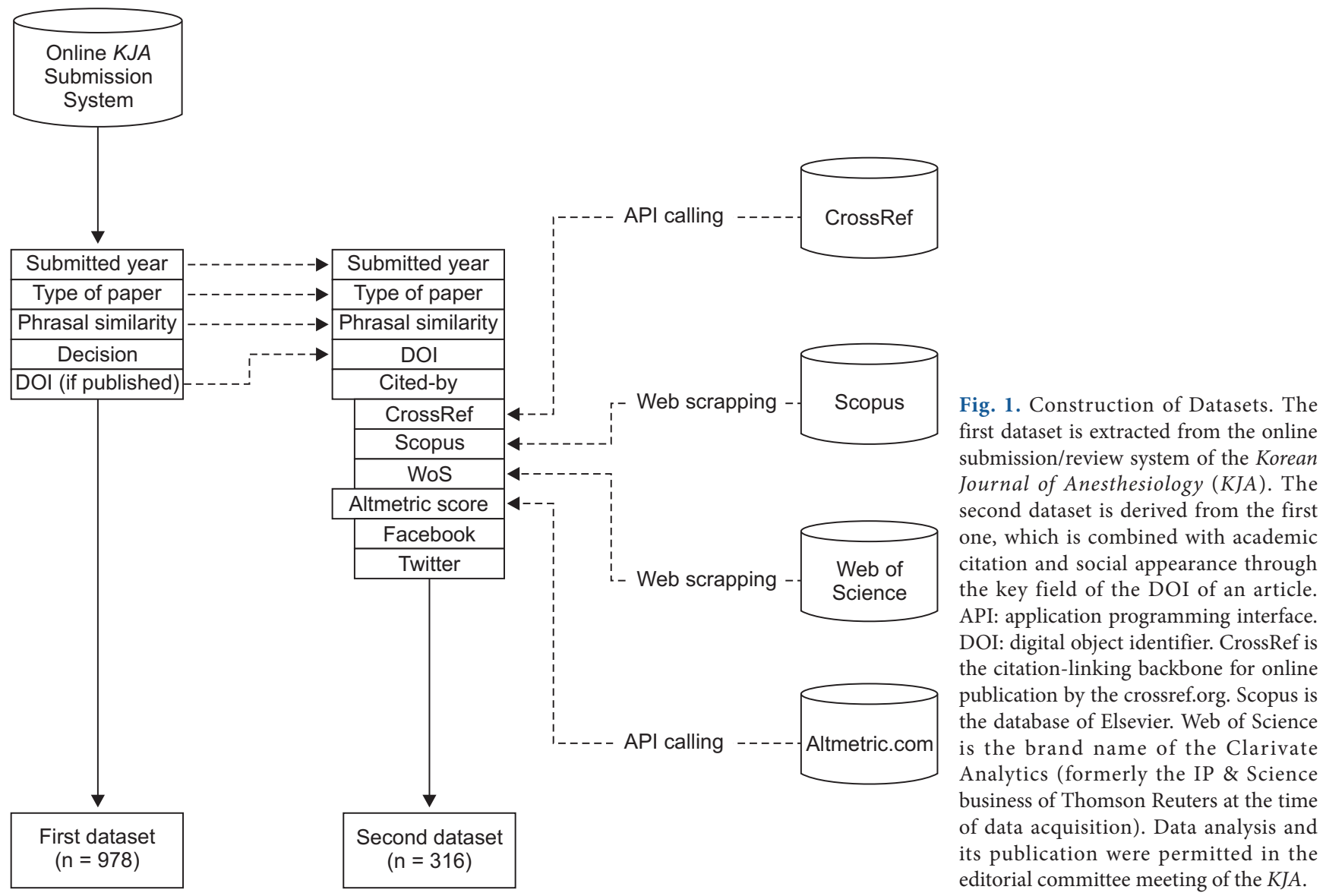

value of a published paper (Fig. 1).

\section{Summary statistics}

The yearly trend of the similarity index of submitted papers was estimated using the first dataset. The similarity index of published papers was also estimated using the second dataset according to whether it had been cited or not. Summary statistics for three cited-by frequency measures and three social values were created.

\section{The similarity index and the value of a submitted paper}

The KJA reviewers and editors were not blinded to the similarity index of the paper, which might influence their opinion during the peer review and decision. Nevertheless, since 2013, the KJA annual refresher course program has educated the reviewers and editors to review a paper irrespective of the similarity index, which has encouraged them to make a decision entirely upon the value of a paper, not upon the similarity index. The probability of acceptance or rejection was partitioned using recursive partitioning analysis. Among the independent vari- ables in the analysis, the similarity index, submission year, and type of the paper were included.

\section{The similarity index and the value of a published paper}

The hidden correlation structure behind the similarity index, publication period, 3 measures of academic value, and 3 measures of social value were estimated using the principal component analysis. On extraction of the principal components, because the 8 variables were on varying scale, the correlation matrix was necessarily applied rather than the covariance matrix.

\section{Open API calling and analytic tool}

The cited-by frequency measures were collected manually from the websites of Scopus and WoS. The cited frequency by CrossRef was obtained with open API call on R statistical software. The number of appearances in a Facebook public wall post and Twitter, and Altmetric.com overall score were obtained with open API call on R. The former was implemented as crossref (Client for Various 'CrossRef' 'APIs'. R package version 0.3.4.) and the latter was implemented as rAltmetric (Retrieves Altmer- 
ics Data For Any Published Paper From Altmetric.com. R package version 0.6). Every Altmetric.com query requires a user key that was authenticated by Altmetric.com. Recursive partitioning analysis was performed using rpart (rpart: Recursive Partitioning and Regression Trees. R package version 4.1-10). R statistical software version 3.2.3 'Wooden Christmas-Tree' (R: A language and environment for statistical computing. R Foundation for Statistical Computing, Vienna, Austria) was used to perform the whole process of data archiving, web retrieval, and statistical analyses. In the current experiment, all statistical analyses were of a purely exploratory nature; therefore, the inferential threshold was not targeted.
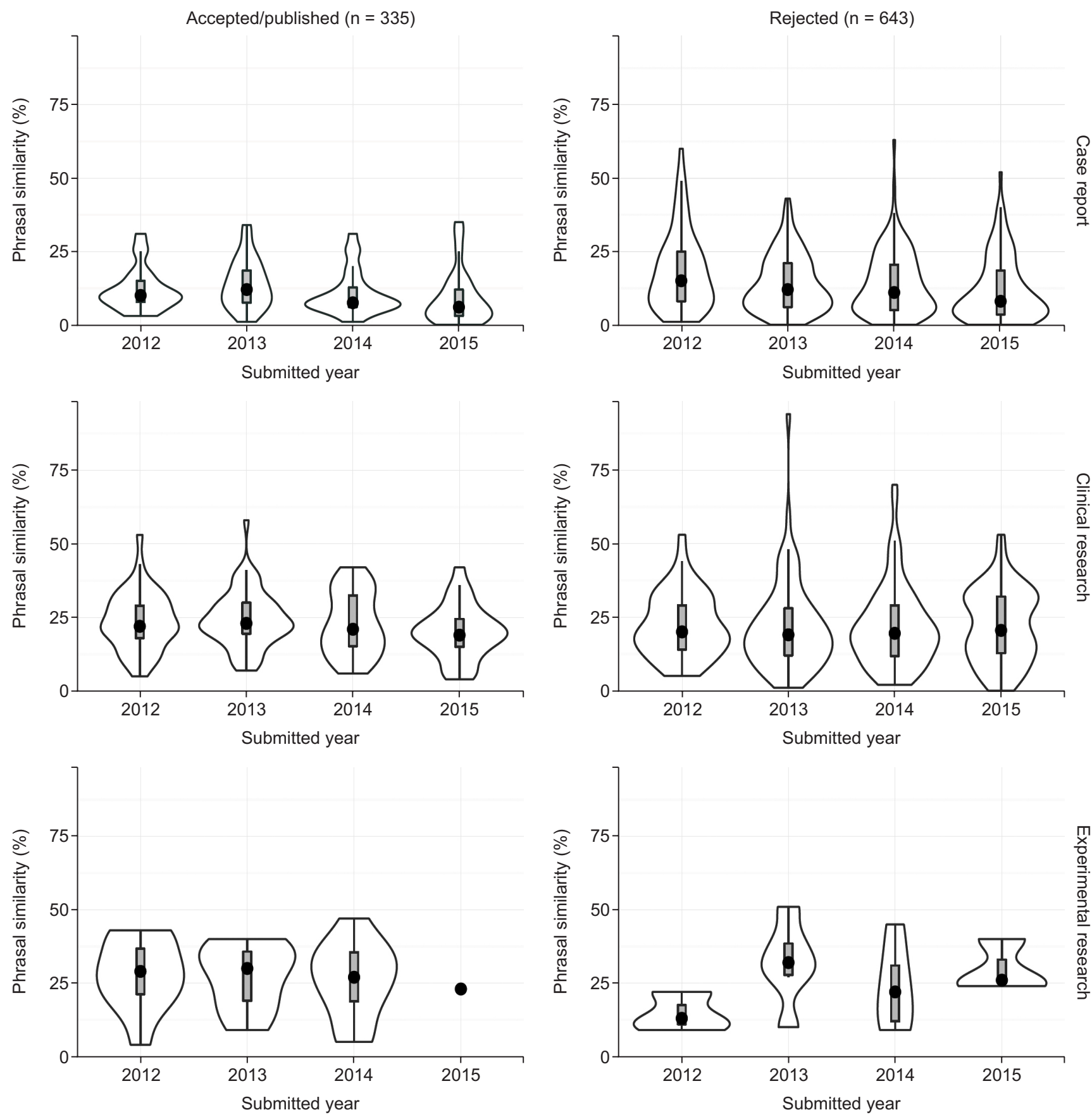

Fig. 2. Distribution of the similarity index in the 978 papers submitted to the Korean Journal of Anesthesiology from 2012 to 2015. In each boxplot, width of violin indicates the kernel density estimate, while borders of a box indicate the $\mathrm{Q}_{1}$ and $\mathrm{Q}_{3}$ with a circle at the median. 


\section{Results}

\section{Summary statistics}

The similarity index approached a mean value of 18 (median value 16, Q1-Q3, 8-26, Table 1), which did not show noticeable changes according to the submission year (Fig. 2). Sixty percent (254/420) of the published papers were cited at least once, while only $42 \%$ (179 of 420 ) of the published papers were mentioned in social media (Table 2).

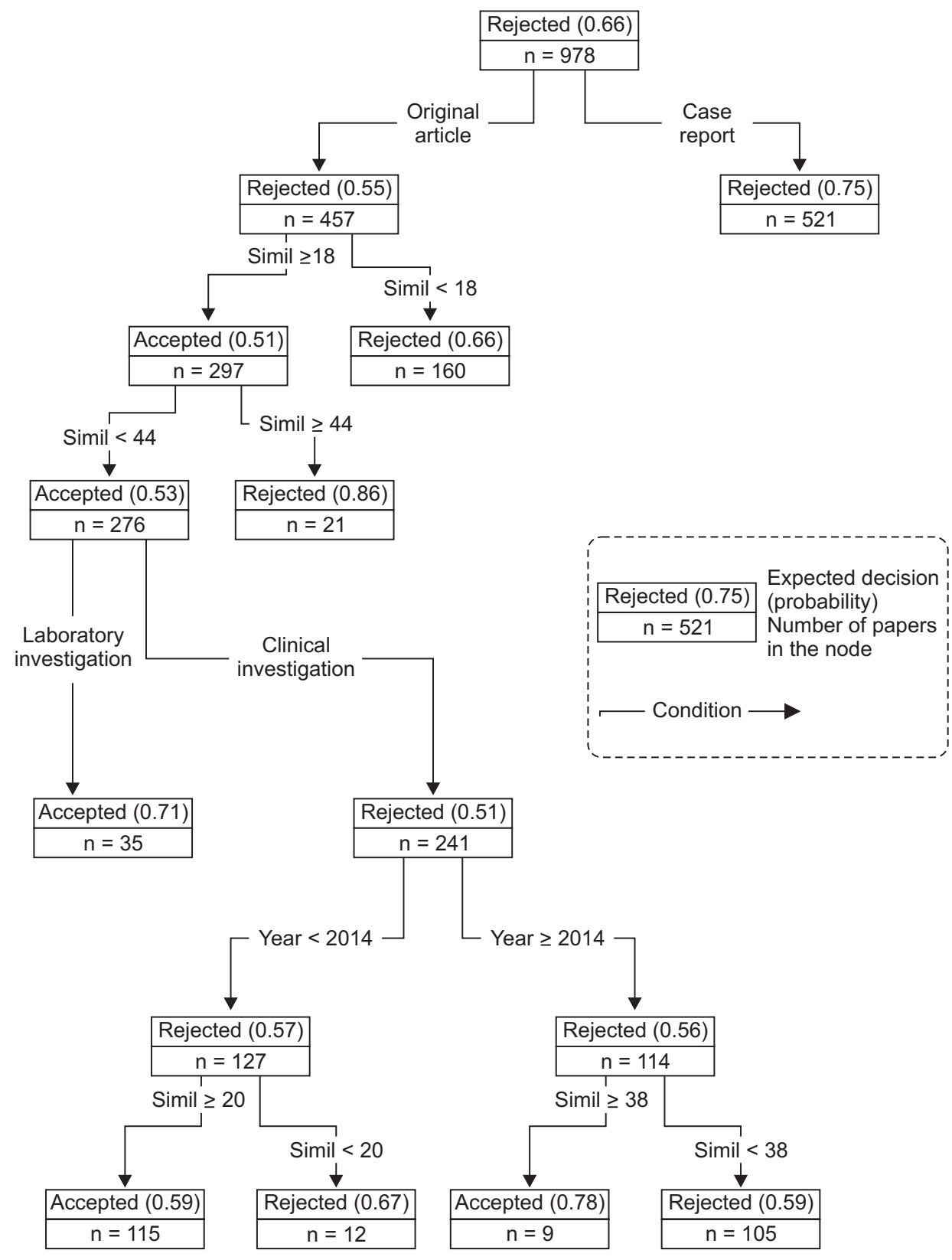

\section{The effect of the similarity index on the value of a submitted paper}

The average rejection rate for submitted papers was 0.66 . Case reports were more frequently rejected than research articles $(0.75$ vs. 0.55$)$. The similarity index caused four splits in the classification tree; research articles having a similarity index less than $18 \%$ were rejected; research articles having a similarity index greater than or equal to $44 \%$ were rejected; articles on clinical research having a similarity index less than $20 \%$ were rejected before the year 2014, and those having a similarity index
Fig. 3. Classification tree for predicting acceptance/rejection of the 978 papers submitted to the Korean Journal of Anesthesiology from 2012 to 2015. Classification is attempted with 3 predetermined variables such as the type of the paper, the submission year, and the similarity index. A recursive partitioning model involves the similarity index, 'simil' in four splits. Each node represents the expected decision, its probability in parenthesis, and the number of papers in the node. Each arrow denotes a branch of the split, in which a condition is represented. To facilitate easy interpretation, a reference node is drawn in a box fenced with a broken line. 
Table 1. Year-wise Trend of the Similarity Index in 978 Submitted Papers in the Korean Journal of Anesthesiology

\begin{tabular}{cccccc}
\hline Submission year & Mean & SD & $\mathrm{Q}_{1}$ & Median & $\mathrm{Q}_{3}$ \\
\hline 2012 & 19 & 12 & 10 & 18 & 28 \\
2013 & 19 & 12 & 9 & 17 & 26 \\
2014 & 18 & 12 & 8 & 16 & 25 \\
2015 & 16 & 12 & 5 & 14 & 25 \\
\hline
\end{tabular}

Table 2. Academic Citation Frequency and Number of Social Media Appearances of the 420 Papers Published by the Korean Journal of Anesthesiology

\begin{tabular}{lcc}
\hline & Never appeared & Appeared \\
\hline Never cited & 102 & 64 \\
Cited & 139 & 115 \\
\hline
\end{tabular}

The term 'Cited' indicates the number of articles cited at least once by the CrossRef, Scopus, and Web of Science. The term 'Appeared' indicates the number of articles having an Altmetric.com overall score $>0$.

less than 38\% were rejected from the year 2014 (Fig. 3). To put it another way, the rejection rate for case reports was not affected by the similarity index. Rejection of research articles having a low similarity index (less than 18\%) accounted for the largest portion of the whole rejection $(106$ papers $=160 \times 0.66)$. Both extremes of the similarity index (less than $18 \%$ or greater than $44 \%)$ led to rejection, irrespective of the submission year. The secondary lowest cutoff values were $20 \%$ and $38 \%$ by the year 2014 as an intersecting point.

\section{The effect of the similarity index on the value of a published paper}

Out of the estimated principal components, we displayed and interpreted only the first three components, whose eigenvalues were $3.00,2.66$, and 0.94 , respectively. These three principal components accounted for almost $85 \%$ of the total variance of the original variables (Table 3 ). The three parameters indicating academic value (CrossRef, Scopus, and WoS) nearly overlapped each other, which was also true for the three parameters indicating social value (Altmetric.com score and appearances in the Facebook wall post and Twitter). The first component indicated that a high (or low) similarity index correlated with a high (or low) value of academic citation, whose loadings were even small. On examination of the second and third component, the similarity index did not have a correlation with either the value of academic citation or with social value. We can see that the third component showed that the higher the publication year (= the more recent the paper), the higher the similarity index. This might reveal that editors gain confidence in accepting a paper,
Table 3. The Hidden Correlation Structure Behind the Submission Year, Similarity Index, Academic Citation, and Social Appearance of the 316 Papers Published by the Korean Journal of Anesthesiology

\begin{tabular}{lccc}
\hline \multicolumn{1}{c}{ Component } & 1 & 2 & 3 \\
\hline Eigenvalue & 3.00 & 2.66 & 0.94 \\
Proportion of variance & 0.38 & 0.33 & 0.12 \\
Cumulative proportion & 0.38 & 0.71 & 0.82 \\
Loadings & & & \\
Publication year* & 0.32 & - & 0.23 \\
Similarity index & -0.18 & - & 0.96 \\
Cited-by: & & & \\
Crossref & -0.52 & - & - \\
Scopus & -0.55 & - & - \\
Web of Science & -0.51 & - & - \\
Altmetric.com: & & & - \\
Overall score & -0.13 & 0.57 & - \\
Facebook & - & 0.55 & - \\
Twitter & -0.10 & 0.58 & Education \\
Labelling & (inverse) age & High social & interest only \\
& effect on & intect & \\
& citation & &
\end{tabular}

*Publication year indicates the year when a paper was published (20122015). Hyphens (-) in place of loadings indicate near-zero values.

independent of the high similarity index.

\section{Discussion}

In our research, in a Korean medical journal, some degree of the similarity index was inevitable, and the permissible range of the similarity index varied depending on the type of the submitted paper. Once published, the similarity index was not related to the value of a paper in the academic or the social aspect.

Our dataset was contaminated by well-understood flaws as the estimated similarity index value was exposed during peer review and final decision. A high similarity index might incline the reviewer and the editor towards relinquishing publication of the paper. Furthermore, we omitted some bibliometric measurements in the analysis, which have been known to be potential candidates that influence the cited-by frequencies of scientific articles [2]. Readers must be aware of the possible expansion of the role of the similarity index in explaining the value of a submitted paper. It should be noted that our finding indicated a correlation rather than causality. Despite the inherent weakness in our dataset and the analysis we have chosen, the result would be worth examining in detail because the dataset registered the full submitted papers since 2012. This is the first published report showing the relationship between the similarity index and the value of a scientific paper.

The highest cutoff value for acceptance of clinical investigation rose to $38 \%$ compared to $20 \%$ during the years before 2014 , which also supported our editorial policy that peer review and a 
decision should be made independent of the similarity index, as mentioned above.

We adopted the cited-by measures as the academic value of a published paper, and then we obtained them from 3 sources. The cited-by measures showed consistent behavior in the principal component analysis, which concluded that the measures from 3 different sources were needless in a research of this nature. The cited-by measures should be interpreted with caution. It must grow with age of the published paper. In the Table 3, the publication year must indicate the inverse age of the paper, which dominates the negative influence on the cited-by measures and the social measure in the first component. Papers published in the year 2015 were enrolled in the analysis, which had a low chance to be cited or to appear in social media likewise. The similarity index showed up twice in the analysis of published papers, being the first in an inverse direction to the publication period and in the same direction to the value of a paper. This suggests that "old papers had a low similarity index and had a high value," in plain English. Second, the direction to the publication period was the same, which may be interpreted as a "time-dependent increase in the similarity index of a published paper." The latter might reflect a gradual change in our editor's standpoint along with the editorial policy that has educated peer reviewers and editors not to reject a paper simply because of a high similarity index. Taken together, we assume that the similarity index varied according to the publication period rather than according to the value of a paper. This is why the first component was named the 'age effect,' and the third component was named the 'education effect.' Independent of these 2 effects, three social media measures accounted for $33 \%$ of total variances.

The Altmetric.com measures account for a wide spectrum of values following the social appearance; composed of social media shares, blog posts, Wikipedia citations, and news stories. ${ }^{1)}$ The KJA has a long history but it is relatively new as an international journal; therefore, appearances in social media are quite rare. Only 179 of the 420 papers appeared in social media as compared with 254 of the 420 papers cited at least once. This discrepancy was also evident on examining the differences in loadings of the first component.

As the academic citation and social appearance shared a unit measurement, count, they were meant to be interpreted by com-

\footnotetext{
${ }^{1)}$ What are Altmetrics? From http://www.altmetric.com/about-altmetrics/ what-are-altmetrics/
}

parison of the absolute values of loadings. The age of an article contributed more to the growth of the academic value than to the growth of the social value (0.5 vs. 0.1$)$. Low contribution of the similarity index, regarding the social value, limited aggressive interpretation. For these reasons, our research did not treat the temporal schema of Tweets (a message sent using Twitter), which can predict high academic citation in an article that appeared on Tweets within the first 3 days of publication [3].

The discovery was made after a cross-sectional investigation of a single medical journal. The finding suggested that the similarity index no longer had an influence on academic citation or social media appearance according to the paper after publication, while the similarity index affected the acceptance/rejection of a submitted paper. Phrases written by authors belonging to a discipline must share a similarity, especially in the case of a non-English native author [4]. Proofreading and intervention to reduce the similarity for finalizing a draft by the editors might play a role in achieving uniform quality of the publication.

Editors are asked to provide a free and transparent exchange of scientific findings, essentially when these findings are related to the article-selection process. With our novel approach to feature the similarity index, medical journal editors will improve their instinct and expand their knowledge about how the similarity index works in decision making for publication and how it propagates beyond its publication. Based on our finding, while the similarity index influences the destiny of a submitted paper in a complicated way depending upon the article type, once the paper is published, the similarity index lacks influence over the academic citation and appearance in social media. Although readers should be aware that our finding was based on a single international journal published by a medical society in Korea, it necessarily concurs with a gleam in an average editor's eye.

\section{Conflict of Interest}

Y. L. was the Editor-in-Chief of Korean Journal of Anesthesiology at the time of research and writing of this manuscript.

\section{ORCID}

Seunghyun Chung, http://orcid.org/0000-0003-4602-2144

Jeunghyuk Lee, http://orcid.org/0000-0001-6947-1669

Younsuk Lee, http://orcid.org/0000-0003-2488-5926

Ha Yeon Park, http://orcid.org/0000-0002-8347-349X

Daehwan Kim, http://orcid.org/0000-0002-3988-0987 


\section{References}

1. Combating plagiarism. Nat Photonics 2009; 3: 237.

2. Haustein S, Costas R, Larivière V. Characterizing social media metrics of scholarly papers: the effect of document properties and collaboration patterns. PLoS One 2015; 10: e0120495.

3. Eysenbach G. Can tweets predict citations? Metrics of social impact based on Twitter and correlation with traditional metrics of scientific impact. J Med Internet Res 2011; 13: e123.

4. Yilmaz I. Plagiarism? No, we're just borrowing better English. Nature 2007; 449: 658. 